\title{
Relationship between body mass index and the expression of hormone receptors or human epidermal growth factor receptor 2 with respect to breast cancer survival
}

\author{
Ye Won Jeon', Su Hwan Kang ${ }^{2}$, Min Ho Park ${ }^{3}$, Woosung Lim ${ }^{4}$, Se Heun Cho ${ }^{5}$ and Young Jin Suh ${ }^{1 *}$
}

\begin{abstract}
Background: The association between body mass index (BMI) at the time of breast cancer diagnosis and the prognosis of breast cancer patients remains controversial. Furthermore, the association between BMI and prognosis with respect to different breast cancer subtypes is not clearly defined.

Methods: We analyzed data from 41,021 invasive breast cancer patients between January 1988 and February 2008 from the Korean Breast Cancer Registry (KBCR) database. Overall survival (OS) and breast cancer-specific survival (BCSS) were analyzed using the Kaplan-Meier method and Cox's proportional hazard regression model among all patients and specific breast cancer subtypes with respect to BMI categories.

Results: A U-shaped association between BMI and mortality was observed in the total cohort. Underweight and obese individuals exhibited worse OS (hazard ratio, 1.23 [95 \% confidence interval \{Cl\}, 1.05 to 1.44] and 1.29 [1.13 to 1.48], respectively) and BCSS (1.26 [1.03 to 1.54] and 1.21 [1.02 to 1.43], respectively) than normal-weight individuals. In the estrogen receptor (ER) and/or progesterone receptor (PR)+/human epidermal growth factor receptor 2 (HER2) - subgroup, obese individuals exhibited worse OS (1.48 [1.18 to 1.85]) and BCSS (1.31 [1.13 to 1.52]) than normal-weight individuals. Conversely, in the ER and PR-/HER2+ subgroup, underweight individuals exhibited worse OS (1.68 [1.12 to 2.47]) and BCSS (1.79 [1.11 to 2.90]) than normal-weight individuals.

Conclusions: We observed a U-shaped relationship between BMI at diagnosis and poor OS and BCSS among all breast cancer patients. However, obesity in the ER and/or PR+/HER2- subgroup and underweight in the ER and PR-/ HER2+ subgroup were poor prognostic factors. Therefore, BMI at diagnosis and breast cancer subtype should be considered simultaneously in various treatment decision processes and surveillance schedules.
\end{abstract}

Keywords: Breast neoplasms, Body mass index, Survival, Estrogen receptor, Progesterone receptor, Human epidermal growth factor receptor 2

\section{Background}

The association between body mass index (BMI) at the time of breast cancer diagnosis and the prognosis of breast cancer patients remains controversial despite many studies, including single institution, multi-center, and population-based studies, meta-analyses, and randomized

\footnotetext{
*Correspondence: yjsuh@catholic.ac.kr

'Department of Surgery, St. Vincent's Hospital, College of Medicine, The Catholic University, 93 Joongboo-Daero Paldal-gu, Suwon 442-723, Kyunggi-do, Republic of Korea

Full list of author information is available at the end of the article
}

controlled trials [1-24]. In many studies, a high BMI at the time of breast cancer diagnosis has been identified as a negative prognostic factor [1-17]. However, several studies have suggested that a low BMI at the time of breast cancer diagnosis correlates with a negative prognosis in breast cancer patients [18-20]. Some investigators have reported a weak or no relationship between BMI and prognosis in breast cancer patients [21-24].

Previous studies have not adequately demonstrated an association between BMI at the time of breast cancer diagnosis and prognosis in breast cancer patients with respect 
to breast cancer subtypes. Recent advances in our understanding of breast cancer biology based on molecular techniques allow us to divide breast cancer into at least four subtypes $[25,26]$. These breast cancer subtypes exhibit different prognoses according to the estrogen receptor (ER), progesterone receptor (PR) and human epidermal growth factor receptor 2 (HER2) expressions. Therefore, it is important to understand the association between BMI and prognosis in the different breast cancer subtypes.

Moreover, there are certain differences between Asian and Western regions with respect to the prevalence of obesity. Although the prevalence of obesity is lower in Asians, the health risks associated with obesity occur at a lower BMI in Asian populations [27-29]. Therefore, an analysis of a large population-based cohort is needed to understand the prognostic significance of obesity in Asian breast cancer patients.

The aim of this study was to investigate the prognostic significance of BMI at the time of breast cancer diagnosis in all breast cancer patients and in each breast cancer subtype by analyzing overall survival (OS) and breast cancerspecific survival (BCSS) using population-based data from the Korean Breast Cancer Registry (KBCR) database.

\section{Methods}

\section{Korean breast cancer registry (KBCR)}

The KBCR database is a web-based, prospectively maintained nationwide database managed by the Korean Breast Cancer Society (KBCS). One hundred and two institutions have voluntarily participated in this registry since 1997 . Before inserting personal information along with various datasets, written informed consent should be mandatory from the patient. From the initial conception of KBCR database, principal investigators from every single institution have agreed on the principles and process of utilizing this database for research purposes. After 2000, an online registration program was implemented, and the database has been actively utilized for various research studies on breast cancer in Korea [18, 30]. Essential registry items include the patient's unique Korean resident registration number, gender, age, the surgical method used, and cancer stage according to the seventh edition of American Joint Committee on Cancer classification [31]. Moreover, data on height, weight, biological status (such as ER, PR, HER2, p53, and Ki67 status), and adjuvant treatment (such as radiotherapy, chemotherapy, and hormonal therapy) are collected as optional items within the KBCR database. The Korean Central Cancer Registry provides mortality data only, and the KBCR does not include information on tumor recurrence.

According to the guidelines of utilizing KBCR database, this study was approved by the institutional Review Board (IRB) of St. Vincent's Hospital, College of Medicine, The Catholic University, where the first author of this article is affiliated (VC14RISI0234).

\section{Patients and follow-up}

In this study, we selected and assessed invasive breast cancer patients who underwent curative surgery between January 1988 and February 2008. To achieve a more accurate analysis, we excluded patients treated with neoadjuvant therapy and patients for whom essential registry data (gender, age, height, weight and cancer stage) and ER/PR status were not available. Patients with distant metastasis at the time of diagnosis were excluded, because distant metastasis is the worst prognostic factor compared with other prognostic factors (such as age, tumor size, histologic grade, lymph node status, adjuvant treatment, BMI, hormone receptor status and HER2 expression) and serves as confounding factor for survival analysis.

The data on the remaining 41,021 patients were included in the final analysis.

All patients were categorized into five subgroups according to the expression of ER, PR and HER2 as follows: (a) ER and/or PR+/HER2-; (b) ER and/or PR+/HER2+; (c) ER and PR-/HER2+; (d) ER and PR-/HER2-; and (e) unknown. All patients for whom ER/PR expression but not HER2 expression information was available were categorized into the unknown group.

Positive staining for ER or PR was defined as the positive staining of $\geq 10 \%$ nuclei in ten high-power fields, and HER2 positivity was defined as $3+$ immunohistochemical (IHC) staining or HER2 gene amplification by fluorescence in situ hybridization (FISH). Cases of 2+ HER2 by IHC without a FISH result were treated as HER2-negative.

Patient survival data, including the date and cause of death, were obtained from the Korean Central Cancer Registry, Ministry of Health and Welfare, Korea.

\section{Statistical analysis}

BMI was calculated by dividing weight $(\mathrm{kg})$ by height $(\mathrm{m})$ squared. The BMI at diagnosis was categorized as normal BMI $\left(18.5-24.9 \mathrm{~kg} / \mathrm{m}^{2}\right)$, underweight BMI $\left(<18.5 \mathrm{~kg} / \mathrm{m}^{2}\right)$, overweight BMI $\left(25.0-29.9 \mathrm{~kg} / \mathrm{m}^{2}\right)$ and obese BMI $\left(\geq 30 \mathrm{~kg} / \mathrm{m}^{2}\right)$ according to the guidelines of the World Health Organization (WHO) [32].

The patient characteristics were compared with respect to BMI category (underweight, normal weight, overweight and obese) using the chi-square test. The chi-square test and analysis of variance (ANOVA) were used to determine differences in the clinicopathological features between groups. With respect to survival analyses, we explored OS and BCSS using data from the KBCR database. OS was defined as the time from the initial diagnosis of primary breast cancer to death from any cause. BCSS was defined as survival until death from breast cancer. Survival curves were estimated using the Kaplan-Meier method. Log-rank tests were performed for the comparison of survival curves. Multivariate analyses were conducted using Cox's proportional-hazard regression models to study the effect 
of BMI at diagnosis on OS and BCSS. The parameters included in the multivariate analysis model were as follows: patient age; tumor size; histologic grade; lymph node status; operation method; adjuvant treatment; ER/PR status and HER2 expression. A $p$ value of less than 0.05 was considered significant. All statistical analyses were performed using the SAS software for Windows (release 9.2; SAS Institute, Cary, NC, USA).

\section{Results}

\section{Patient characteristics}

For the 41,021 patients included in our analysis, the mean age at breast cancer diagnosis was 48 years (range, 18 to 93). The baseline characteristics are presented in Table 1, stratified according to BMI categories. The BMI categories revealed a significant association with known breast cancer prognostic factors. The median age at diagnosis for obese patients was significantly older than underweight patients $(p<0.001)$. Obese patients had larger tumors $(p<0.001)$, high frequencies of axillary lymph node metastasis $(p<0.001)$, histologically high-grade lesions $(p=0.003)$, and negative ER and PR $(p=0.002)$ and HER2 expression $(p=0.004)$ compared with underweight patients.

Breast cancer subgroups categorized according to the expression of ER, PR and HER2 exhibited a significant association with BMI categories $(p<0.001)$. The ER and/or PR+/HER2- and ER and PR-/HER2- subtypes were more prevalent in the obese BMI category. However, the ER and/or PR+/HER2+ subtype was more prevalent in the underweight BMI category.

\section{Overall survival and breast cancer-specific survival}

A total of 4468 deaths from any cause and 2824 deaths from breast cancer were observed over a median followup time of 92 months after diagnosis, with a maximum follow-up of 300 months.

After adjusting for poor prognostic factors, such as tumor size, axillary lymph node metastasis, histologic grade, ER, PR and HER2 expression, a U-shaped association between BMI and mortality was observed in the total cohort (Table 2). Compared with patients in the normal BMI category, those in the underweight BMI category exhibited significantly worse OS (adjusted hazard ratio [HR] 1.23, $95 \%$ confidence interval [CI] 1.05 to 1.44 , $p=0.0118$ ), as did those in the obese BMI category (adjusted HR 1.29, $95 \%$ CI 1.13 to 1.48, $p=0.0002$ ). Patients in the underweight BMI category (adjusted HR 1.26, $95 \%$ CI 1.03 to $1.54, p=0.0219$ ) and the obese BMI category (adjusted HR 1.21, $95 \%$ CI 1.02 to $1.43, p=0.0321$ ) exhibited significantly worse BCSS compared with those in the normal BMI category.

In the ER and/or PR+/HER2- subgroup, patients in the obese BMI category exhibited significantly worse
OS (adjusted HR 1.48, $95 \%$ CI 1.18 to $1.85, p=0.0006$ ) and BCSS (adjusted HR 1.31, $95 \%$ CI 1.13 to $1.52, p=0.0003$ ) compared with those in the normal BMI category (Table 3). However, no significant difference was observed in the OS $(p=0.1269)$ and BCSS $(p=0.2684)$ rate between patients in the normal BMI category and the underweight BMI category. Conversely, in the ER and PR-/HER2+ subgroup, patients in the underweight BMI category exhibited significantly worse OS (adjusted HR 1.67, $95 \%$ CI 1.12 to 2.47, $p=0.0113$ ) and BCSS (adjusted HR 1.79, $95 \%$ CI 1.11 to $2.90, p=0.0179$ ) compared with those in the normal BMI category. However, obese BMI was not associated with decreased OS $(p=0.4247)$ or BCSS $(p=0.5683)$ in the ER and PR-/HER2+ subgroup. In the ER and/or PR+/HER2+ and ER and PR-/HER2- subgroups, BMI categories did not exhibit a significant association with OS and BCSS. In the unknown subgroup, patients in the obese BMI category exhibited significantly worse OS (adjusted HR 1.36, 95 \% CI 1.04 to $1.79, p=0.0261$ ) but not worse BCSS (adjusted HR 1.36, $95 \%$ CI 0.97 to $1.90, p=0.0747$ ) compared with those in the normal BMI category.

\section{Discussion}

In our total cohort analysis, underweight and obese breast cancer patients exhibited significantly poorer OS and BCSS compared with normal BMI category breast cancer patients, suggesting a U-shaped relationship, as has been previously suggested $[7,13,14,17]$. This is the largest study to suggest that breast cancer patients with a normal BMI range at diagnosis exhibit the most favorable breast cancer outcomes.

Similar to our finding, a previous study using the KBCR database demonstrated that underweight $\mathrm{BMI}$ is an independent negative prognostic factor for OS and BCSS after adjustment. However, in a previous study, neither obese patients nor overweight patients exhibited significant differences in OS and BCSS compared with normal-weight patients [18]. Two studies using the KBCR database reported different results for the prognostic significance of obesity in breast cancer patients. A potential explanation for the differing results is that more recent breast cancer patients (between 2007 and 2008) were included our study. Because an increase in overweight BMI and obesity has been noted in South Korean adults [33], our study, which included more recent breast cancer patients, may provide a more accurate analysis of the prognostic significance of obesity in Korean breast cancer patients.

This study is the first to further explore results with respect to both BMI at diagnosis and the four breast cancer subtypes, enabling better identification of women at highest risk of poor outcomes.

In the ER and/or PR+/HER2- subgroup, obese breast cancer patients exhibited significantly worse OS and BCSS 
Table 1 General characteristics of KBCS breast cancer subjects, overall and by BMI categories

\begin{tabular}{|c|c|c|c|c|c|c|}
\hline \multirow[t]{2}{*}{ Characteristic } & \multirow[t]{2}{*}{ Overall } & \multirow{2}{*}{$\begin{array}{l}\text { Underweight BMI } \\
<18.5 \mathrm{~kg} / \mathrm{m}^{2}\end{array}$} & \multirow{2}{*}{$\begin{array}{l}\text { Normal BMl } \\
18.5-24.9 \mathrm{~kg} / \mathrm{m}^{2}\end{array}$} & \multirow{2}{*}{$\begin{array}{l}\text { Overweight BMI } \\
25-29.9 \mathrm{~kg} / \mathrm{m}^{2}\end{array}$} & \multirow{2}{*}{$\begin{array}{l}\text { Obese BMl } \\
\geq 30 \mathrm{~kg} / \mathrm{m}^{2}\end{array}$} & \multirow[t]{2}{*}{$p$ value } \\
\hline & & & & & & \\
\hline No. of subjects & 41021 & 1387 & 27519 & 10483 & 1632 & \\
\hline \multicolumn{7}{|l|}{ Age (years) } \\
\hline mean(SD) & $48(10)$ & $42(10)$ & $47(10)$ & $52(10)$ & $53(11)$ & \multirow[t]{2}{*}{$<0.0001$} \\
\hline Median(Range) & $47(18-93)$ & $41(19-87)$ & $46(18-93)$ & $51(19-90)$ & $53(22-87)$ & \\
\hline$\leq 35$ & $3737(9.11)$ & $390(28.12)$ & 2793(10.15) & $483(4.61)$ & $71(4.35)$ & \multirow[t]{2}{*}{$<0.0001$} \\
\hline$>35$ & $37284(90.89)$ & $997(71.88)$ & 24726(89.85) & 10000(95.39) & 1561(95.65) & \\
\hline \multicolumn{7}{|l|}{ Tumor size $(\mathrm{cm})$} \\
\hline $0-2$ & $21931(53.46)$ & $827(59.63)$ & $15331(55.71)$ & $5083(48.49)$ & $690(42.28)$ & \multirow[t]{2}{*}{$<0.0001$} \\
\hline$>2$ & $19090(46.54)$ & $560(40.37)$ & 12188(44.29) & $5400(51.51)$ & $942(57.72)$ & \\
\hline \multicolumn{7}{|l|}{ Axillary lymph node metastasis } \\
\hline Negative & $25205(61.44)$ & $916(66.04)$ & $17205(62.52)$ & 6153(58.70) & $931(57.05)$ & \multirow[t]{2}{*}{$<0.0001$} \\
\hline Positive & 15816(38.56) & 471(33.96) & 10314(37.48) & $4330(41.30)$ & $701(42.95)$ & \\
\hline \multicolumn{7}{|l|}{ Operation method } \\
\hline Mastectomy & $23362(56.95)$ & $759(54.72)$ & 15361(55.82) & 6254(59.66) & $988(60.54)$ & \multirow[t]{2}{*}{$<0.0001$} \\
\hline Conserving surgery & $17659(43.05)$ & $628(45.28)$ & 12158(44.18) & $4229(40.34)$ & 644(39.46) & \\
\hline \multicolumn{7}{|l|}{ ER/PR expression } \\
\hline ER and/or PR Positive & $28166(68.66)$ & $970(69.94)$ & 19065(69.28) & $7040(67.16)$ & $1091(66.85)$ & \multirow[t]{2}{*}{0.0002} \\
\hline ER and PR Negative & $12855(31.34)$ & $417(30.06)$ & 8454(30.72) & $3443(32.84)$ & $541(33.15)$ & \\
\hline \multicolumn{7}{|l|}{ HER 2 expression } \\
\hline Negative & $28530(69.55)$ & $955(68.85)$ & 18995(69.03) & $7401(70.60)$ & 1179(72.24) & \multirow[t]{3}{*}{0.0004} \\
\hline Positive & $8005(19.51)$ & 265(19.11) & $5541(20.14)$ & 1917(18.29) & $282(17.28)$ & \\
\hline Unknown & $4486(10.94)$ & 167(12.04) & 2983(10.84) & 1165(11.11) & $171(10.48)$ & \\
\hline \multicolumn{7}{|l|}{ Subtype } \\
\hline ER and/or PR + and HER 2 - & $21094(51.42)$ & $706(50.90)$ & $14179(51.52)$ & $5363(51.16)$ & $846(51.84)$ & \multirow[t]{5}{*}{$<0.0001$} \\
\hline ER and/or PR + and HER $2+$ & $4118(10.04)$ & 144(10.38) & 2909(10.57) & $929(8.86)$ & 136(8.33) & \\
\hline ER and PR - and HER $2+$ & $3887(9.48)$ & $121(8.72)$ & $2632(9.56)$ & $988(9.42)$ & 146(8.95) & \\
\hline ER and PR - and HER 2 -- & $7436(18.13)$ & 249(17.95) & $4816(17.50)$ & 2038(19.44) & $333(20.40)$ & \\
\hline Unknown & $4486(10.94)$ & 167(12.04) & 2983(10.84) & 1165(11.11) & $171(10.48)$ & \\
\hline \multicolumn{7}{|l|}{ Histologic grade } \\
\hline Low (grade1 - 2) & 20043(48.86) & $690(49.75)$ & 13495(49.04) & $5050(48.17)$ & $808(49.51)$ & \multirow[t]{3}{*}{0.0032} \\
\hline High (Grade 3) & $15890(38.74)$ & 498(35.90) & 10560(38.37) & 4186(39.93) & $646(39.58)$ & \\
\hline Unknown & $5088(12.4)$ & 199(14.35) & $3464(12.59)$ & $1247(11.90)$ & 178(10.91) & \\
\hline \multicolumn{7}{|l|}{ Adjuvant chemotherapy } \\
\hline Yes & $28812(70.24)$ & $891(64.24)$ & 19289(70.09) & $7477(71.33)$ & 1155(70.77) & $<0.0001$ \\
\hline No & $9682(23.6)$ & 409(29.49) & 6578(23.90) & $2320(22.13)$ & $375(22.98)$ & \\
\hline Unknown & $2527(6.16)$ & $87(6.27)$ & 1652(6.00) & $686(6.54)$ & $102(6.25)$ & \\
\hline Adjuvant hormonal & & & & & & \\
\hline Yes & $26265(64.03)$ & $856(61.72)$ & $17723(64.40)$ & $6651(63.45)$ & $1035(63.42)$ & 0.2424 \\
\hline No & 12782(31.16) & 463(33.38) & $8501(30.89)$ & $3300(31.48)$ & $518(31.74)$ & \\
\hline Unknown & 1974(4.81) & $68(4.90)$ & $1295(4.71)$ & $532(5.07)$ & $79(4.84)$ & \\
\hline
\end{tabular}

compared with normal and underweight BMI breast cancer patients. Previous studies have demonstrated that obesity is associated with an increase in OS or
BCSS in patients with ER and/or PR positive breast cancer but is not in patients with ER and PR negative breast cancer $[1,4,10,11,14]$. Several hypotheses may 
Table 2 Cox's proportional hazard regression model for overall survival (OS) and breast cancer specific survival (BCSS)

\begin{tabular}{|c|c|c|c|c|c|c|c|c|c|c|c|c|}
\hline \multirow[t]{2}{*}{ Characteristic } & \multicolumn{6}{|c|}{ Overall survival } & \multicolumn{6}{|c|}{ Breast cancer specific survival } \\
\hline & Alive & Death & $\mathrm{HR}(95 \% \mathrm{Cl})$ & $p$ value & Adjust HR (95 \% Cl) & $p$ value & Alive & Death & $\mathrm{HR}(95 \% \mathrm{Cl})$ & $p$ value & Adjust HR (95 \% Cl) & $p$ value \\
\hline No. of participants & 36553 & 4468 & & & & & 98197 & 2824 & & & & \\
\hline \multicolumn{13}{|l|}{ Age (years) } \\
\hline$\leq 35$ & $3330(9.11)$ & 407(9.11) & 1.00 & & & & $3479(9.11)$ & 258(9.14) & 1.00 & & & \\
\hline$>35$ & $33223(90.89)$ & $4061(90.89)$ & $1.04(0.94-1.15)$ & 0.4945 & & & $34718(90.89)$ & 2566(90.86) & 1.03(0.91-1.17) & 0.6453 & & \\
\hline \multicolumn{13}{|l|}{ Tumor size $(\mathrm{cm})$} \\
\hline $0-2$ & 20608(56.38) & 1323(29.61) & 1.00 & & 1.00 & & $21185(55.46)$ & $746(26.42)$ & 1.00 & & 1.00 & \\
\hline$>2$ & $15945(43.62)$ & $3145(70.39)$ & $2.74(2.57-2.92)$ & $<0.0001$ & $1.70(1.58-1.82)$ & $<0.0001$ & $17012(44.54)$ & 2078(73.58) & $3.16(2.91-3.44)$ & $<0.0001$ & $1.77(1.61-1.93)$ & $<0.0001$ \\
\hline \multicolumn{13}{|c|}{ Axillary lymph node metastasis } \\
\hline Negative & $23731(64.92)$ & 1474(32.99) & 1.00 & & 1.00 & & $24439(63.98)$ & $766(27.12)$ & 1.00 & & 1.00 & \\
\hline Positive & $12822(35.08)$ & 2994(67.01) & $3.43(3.22-3.65)$ & $<0.0001$ & $2.95(2.75-3.16)$ & $<0.0001$ & $13758(36.02)$ & $2058(72.88)$ & $4.42(4.07-4.80)$ & $<0.0001$ & $3.50(3.20-3.84)$ & $<0.0001$ \\
\hline \multicolumn{13}{|l|}{ Operation method } \\
\hline Mastectomy & 19830(54.25) & 3532(79.05) & 1.00 & & 1.00 & & $21094(55.22)$ & 2268(80.31) & 1.00 & & 1.00 & \\
\hline Conserving surgery & $16723(45.75)$ & $936(20.95)$ & $0.37(0.34-0.39)$ & $<0.0001$ & $0.55(0.51-0.59)$ & $<0.0001$ & 17103(44.78) & $556(19.69)$ & $0.34(0.31-0.37)$ & $<0.0001$ & $0.55(0.50-0.61)$ & $<0.0001$ \\
\hline \multicolumn{13}{|l|}{ ER/PR expression } \\
\hline ER and/or PR Positive & $25647(70.16)$ & 2519(56.38) & 1.00 & & 1.00 & & $26629(69.71)$ & $1537(54.43)$ & 1.00 & & 1.00 & \\
\hline ER and PR Negative & 10906(29.84) & 1949(43.62) & $1.75(1.65-1.86)$ & $<0.0001$ & $1.55(1.42-1.70)$ & $<0.0001$ & 11568(30.29) & $1287(45.57)$ & $1.87(1.73-2.01)$ & $<0.0001$ & $1.56(1.40-1.74)$ & $<0.0001$ \\
\hline \multicolumn{13}{|l|}{ HER 2 expression } \\
\hline Negative & 26098(71.40) & $2432(54.43)$ & 1.00 & & 1.00 & & $27018(70.73)$ & 1512(53.54) & 1.00 & & 1.00 & \\
\hline Positive & $7040(19.26)$ & $965(21.59)$ & $1.42(1.32-1.53)$ & $<0.0001$ & $1.11(1.03-1.20)$ & 0.0061 & 7402(19.38) & $603(21.35)$ & $1.41(1.29-1.55)$ & $<0.0001$ & 1.08(0.98-1.19) & 0.1128 \\
\hline Unknown & $3415(9.34)$ & 1071(23.97) & $2.23(2.07-2.41)$ & $<0.0001$ & $1.70(1.58-1.84)$ & $<0.0001$ & $3777(9.89)$ & $709(25.10)$ & $2.45(2.23-2.68)$ & $<0.0001$ & $1.91(1.74-2.11)$ & $<0.0001$ \\
\hline \multicolumn{13}{|l|}{ BMI calssification } \\
\hline Underweight BMI & $1229(3.36)$ & 158(3.54) & $1.16(0.98-1.36)$ & 0.0784 & $1.23(1.05-1.44)$ & 0.0118 & 1284(3.36) & 103(3.65) & 1.18(0.97-1.44) & 0.0974 & $1.26(1.03-1.54)$ & 0.0219 \\
\hline Normal BMI & $24766(67.75)$ & 2753(61.62) & 1.00 & & 1.00 & & $25774(67.48)$ & $1745(61.79)$ & 1.00 & & 1.00 & \\
\hline Overweight BMI & $9164(25.07)$ & 1319(29.52) & $1.26(1.18-1.35)$ & $<0.0001$ & $1.15(1.07-1.23)$ & $<0.0001$ & $9651(25.27)$ & $832(29.46)$ & $1.25(1.15-1.36)$ & $<0.0001$ & $1.13(1.04-1.22)$ & 0.0052 \\
\hline Obese BMI & 1394(3.81) & 238(5.33) & $1.51(1.32-1.72)$ & $<0.0001$ & $1.29(1.13-1.48)$ & 0.0002 & 1488(3.90) & 144(5.10) & $1.42(1.19-1.68)$ & $<0.0001$ & $1.21(1.02-1.43)$ & 0.0321 \\
\hline \multicolumn{13}{|l|}{ Histologic grade } \\
\hline Low (grade1 - 2) & $18316(50.11)$ & 1727(38.65) & 1.00 & & 1.00 & & $19002(49.75)$ & $1041(36.86)$ & 1.00 & & 1.00 & \\
\hline High (Grade 3) & $13631(37.29)$ & $2259(50.56)$ & $1.73(1.62-1.84)$ & $<0.0001$ & $1.32(1.23-1.41)$ & $<0.0001$ & $14401(37.70)$ & $1489(52.73)$ & $1.86(1.72-2.02)$ & $<0.0001$ & $1.36(1.25-1.48)$ & $<0.0001$ \\
\hline Unknown & $4606(12.60)$ & 482(10.79) & $1.02(0.92-1.13)$ & 0.6849 & $1.04(0.94-1.15)$ & 0.494 & 4794(12.55) & 294(10.41) & 1.04(0.92-1.19) & 0.5269 & $1.08(0.95-1.23)$ & 0.2561 \\
\hline \multicolumn{13}{|l|}{ Adjuvant chemotherapy } \\
\hline Yes & 25339(69.32) & $3473(77.73)$ & 1.00 & & 1.00 & & $26475(69.31)$ & $2337(82.75)$ & 1.00 & & 1.00 & \\
\hline No & $9002(24.63)$ & $680(15.22)$ & $0.58(0.53-0.63)$ & $<0.0001$ & $1.41(1.29-1.55)$ & $<0.0001$ & 9354(24.49) & $328(11.61)$ & $0.42(0.37-0.47)$ & $<0.0001$ & $1.12(0.99-1.28)$ & 0.0753 \\
\hline
\end{tabular}


Table 2 Cox's proportional hazard regression model for overall survival (OS) and breast cancer specific survival (BCSS) (Continued)

\begin{tabular}{|c|c|c|c|c|c|c|c|c|c|c|c|c|}
\hline Unknown & $2212(6.05)$ & $315(7.05)$ & $1.02(0.91-1.14)$ & 0.7874 & $1.09(0.97-1.24)$ & 0.1448 & $2368(6.20)$ & 159(5.63) & $0.75(0.64-0.88)$ & 0.0005 & $0.79(0.67-0.94)$ & 0.0062 \\
\hline \multicolumn{13}{|c|}{ Adjuvant hormonal } \\
\hline Yes & 23785(65.07) & $2480(55.51)$ & 1.00 & & 1.00 & & $24743(64.78)$ & 1522(53.90) & 1.00 & & 1.00 & \\
\hline No & $11000(30.09)$ & 1782(39.88) & $1.53(1.44-1.63)$ & $<0.0001$ & 1.07(0.98-1.17) & 0.1399 & 11604(30.38) & 1178(41.71) & $1.63(1.51-1.76)$ & $<0.0001$ & $1.12(1.01-1.25)$ & 0.0414 \\
\hline Unknown & $1768(4.84)$ & $206(4.61)$ & $1.13(0.98-1.30)$ & 0.0920 & $1.02(0.88-1.19)$ & 0.7627 & 1850(4.84) & 124(4.39) & $1.10(0.92-1.32)$ & 0.3058 & $1.04(0.86-1.25)$ & 0.6977 \\
\hline
\end{tabular}

Abbreviations: OS overall survival, $B C S S$ breast cancer specific survival, $H R$ hazard ratio

Data presented as $\mathrm{n}(\%)$ and $\mathrm{HR}(95 \% \mathrm{Cl})$

HRs are unadjusted or adjusted based on Cox's proportional-hazard regression models

Patient age; tumor size; histologic grade; lymph node status; operation method; adjuvant treatment; ER/PR status and HER2 expression included in the multivariate analysis model 
Table 3 Cox's proportional hazard regression model for overall survival (OS) and breast cancer specific survival (BCSS) among breast cancer subtypes

\begin{tabular}{|c|c|c|c|c|c|c|c|c|c|c|c|c|}
\hline \multirow[t]{2}{*}{ Subtype } & \multicolumn{6}{|c|}{ Overall survival } & \multicolumn{6}{|c|}{ Breast cancer specific survival } \\
\hline & Alive & Death & HR $(95 \%$ Cl) & $p$ value & Adjust HR (95 \% Cl) & $p$ value & Alive & Death & HR $(95 \%$ Cl) & $p$ value & Adjust HR (95 \% Cl) & $p$ value \\
\hline \multicolumn{13}{|c|}{ ER and/or PR + and HER 2 - } \\
\hline No. of participants & 19674 & 1420 & & & & & 20248 & 846 & & & & \\
\hline Underweight BMI & 659(3.35) & $47(3.31)$ & $1.17(0.87-1.57)$ & 0.2902 & $1.26(0.94-1.69)$ & 0.1269 & 678(3.35) & $28(3.31)$ & $1.17(0.80-1.71)$ & 0.4239 & $1.24(0.85-1.82)$ & 0.2684 \\
\hline Normal BMl & 13352(67.87) & $827(58.24)$ & 1.00 & & 1.00 & & $13686(67.59)$ & $493(58.27)$ & 1.00 & & 1.00 & \\
\hline Overweight BMl & $4901(24.91)$ & $462(32.54)$ & $1.48(1.32-1.66)$ & $<0.0001$ & $1.32(1.18-1.48)$ & $<0.0001$ & $5084(25.11)$ & 279(32.98) & $1.49(1.29-1.73)$ & 0.0035 & $1.30(0.96-1.76)$ & 0.0874 \\
\hline Obese BMI & $762(3.87)$ & $84(5.92)$ & $1.74(1.39-2.18)$ & $<0.0001$ & $1.48(1.18-1.85)$ & 0.0006 & $800(3.95)$ & $46(5.44)$ & $1.57(1.16-2.12)$ & $<0.0001$ & $1.31(1.13-1.52)$ & 0.0003 \\
\hline \multicolumn{13}{|c|}{ ER and/or PR + and HER $2+$} \\
\hline No. of participants & 3691 & 427 & & & & & 3851 & 267 & & & & \\
\hline Underweight BMI & 130(3.52) & $14(3.28)$ & $0.95(0.56-1.62)$ & 0.8463 & $1.07(0.63-1.83)$ & 0.802 & 139(3.61) & $5(1.87)$ & $0.54(0.22-1.31)$ & 0.1737 & $0.61(0.25-1.48)$ & 0.2705 \\
\hline Normal BMI & 2612(70.77) & $297(69.56)$ & 1.00 & & 1.00 & & 2726(70.79) & 183(68.54) & 1.00 & & 1.00 & \\
\hline Overweight BMl & $827(22.41)$ & 102(23.89) & $1.06(0.85-1.33)$ & 0.5960 & $1.02(0.81-1.28)$ & 0.875 & $856(22.23)$ & $73(27.34)$ & $1.24(0.95-1.63)$ & 0.1177 & $1.18(0.90-1.55)$ & 0.2251 \\
\hline Obese BMI & $122(3.31)$ & $14(3.28)$ & $1.05(0.61-1.79)$ & 0.8608 & $0.94(0.55-1.61)$ & 0.8193 & 130(3.38) & $6(2.25)$ & $0.72(0.32-1.62)$ & 0.4277 & $0.62(0.27-1.39)$ & 0.2449 \\
\hline \multicolumn{13}{|c|}{ ER and PR - and HER 2 + } \\
\hline No. of participants & 3349 & 538 & & & & & 3551 & 336 & & & & \\
\hline Underweight BMI & $94(2.81)$ & $27(5.02)$ & $1.79(1.21-2.65)$ & 0.0034 & $1.67(1.12-2.47)$ & 0.0113 & 103(2.90) & $18(5.36)$ & $1.88(1.16-3.05)$ & 0.0099 & 1.79(1.11-2.90) & 0.0179 \\
\hline Normal BMl & 2288(68.32) & $344(63.94)$ & 1.00 & & 1.00 & & 2418(68.09) & 214(63.69) & 1.00 & & 1.00 & \\
\hline Overweight BMl & $847(25.29)$ & $141(26.21)$ & $1.09(0.90-1.33)$ & 0.3926 & 0.94(0.77-1.14) & 0.5093 & $900(25.34)$ & $88(26.19)$ & $1.09(0.85-1.40)$ & 0.4921 & 0.93(0.73-1.19) & 0.5715 \\
\hline Obese BMI & $120(3.58)$ & $26(4.83)$ & $1.38(0.93-2.06)$ & 0.1132 & $1.18(0.79-1.76)$ & 0.4247 & 130(3.66) & $16(4.76)$ & $1.35(0.81-2.24)$ & 0.2496 & $1.16(0.70-1.93)$ & 0.5683 \\
\hline \multicolumn{13}{|c|}{ ER and PR - and HER 2 - } \\
\hline No. of participants & 6424 & 1012 & & & & & 6770 & 666 & & & & \\
\hline Underweight BMI & 215(3.35) & 34(3.36) & $1.09(0.77-1.54)$ & 0.6222 & $1.19(0.84-1.68)$ & 0.3333 & 225(3.32) & $24(3.60)$ & $1.14(0.76-1.72)$ & 0.5347 & $1.27(0.84-1.92)$ & 0.2606 \\
\hline Normal BMl & $4206(65.47)$ & $610(60.28)$ & 1.00 & & 1.00 & & $4402(65.02)$ & $414(62.16)$ & 1.00 & & 1.00 & \\
\hline Overweight BMl & 1727(26.88) & $311(30.73)$ & $1.20(1.05-1.38)$ & 0.0080 & $1.07(0.93-1.23)$ & 0.3301 & 1849(27.31) & 189(28.38) & $1.07(0.90-1.27)$ & 0.4398 & 0.93(0.79-1.11) & 0.4320 \\
\hline Obese BMI & $276(4.30)$ & $57(5.63)$ & $1.37(1.04-1.80)$ & 0.0234 & $1.18(0.90-1.55)$ & 0.2395 & $294(4.34)$ & $39(5.86)$ & $1.38(0.99-1.91)$ & 0.0571 & $1.16(0.84-1.61)$ & 0.3749 \\
\hline \multicolumn{13}{|l|}{ Unknown } \\
\hline No. of participants & 3415 & 1071 & & & & & 3777 & 709 & & & & \\
\hline Underweight BMI & 131(3.84) & $36(3.36)$ & $0.98(0.70-1.37)$ & 0.8923 & $1.11(0.79-1.55)$ & 0.5528 & 139(3.68) & 28(3.95) & $1.16(0.79-1.70)$ & 0.44 & $1.29(0.87-1.89)$ & 0.2010 \\
\hline Normal BMI & 2308(67.58) & $675(63.03)$ & 1.00 & & 1.00 & & $2542(67.30)$ & $441(62.20)$ & 1.00 & & 1.00 & \\
\hline Overweight BMl & $862(25.24)$ & $303(28.29)$ & $1.18(1.03-1.35)$ & 0.0153 & $1.12(0.98-1.28)$ & 0.1042 & $962(25.47)$ & $203(28.63)$ & $1.20(1.02-1.42)$ & 0.0283 & $1.15(0.97-1.36)$ & 0.1030 \\
\hline Obese BMI & 114(3.34) & $57(5.32)$ & $1.61(1.23-2.11)$ & 0.0005 & $1.36(1.04-1.79)$ & 0.0261 & 134(3.55) & $37(5.22)$ & $1.56(1.11-2.18)$ & 0.0099 & $1.36(0.97-1.90)$ & 0.0747 \\
\hline
\end{tabular}

Abbreviations: OS overall survival, $B C S S$ breast cancer specific survival, $H R$ hazard ratio

Data presented as $\mathrm{n}(\%)$ and $\mathrm{HR}(95 \% \mathrm{Cl})$

HRs are unadjusted or adjusted based on Cox's proportional-hazard regression models

Patient age; tumor size; histologic grade; lymph node status; operation method; adjuvant treatment; ER/PR status and HER2 expression included in the multivariate analysis model 
explain why obese patients with HR-positive breast cancer exhibit worse survival [8,34-36]. Obesity is related to the increased peripheral conversion of androgenic precursors to estradiol due to increased aromatase enzyme activity from large amounts of adipose tissue and is also related to decreased sex hormone-binding globulin [8, 34]. Additionally, obesity can increase insulin and insulin-like growth factors and obesity-related regulatory proteins, such as leptin and adiponectin $[35,36]$. As a result, high circulating bioavailable estrogen, growth factors and regulatory proteins could have a carcinogenic effect, promoting tumor growth and progression, in breast cancer cells expressing the estrogen receptor.

In contrast to the ER and/or PR+/HER2- subgroup, underweight breast cancer patients exhibit significantly worse OS and BCSS compared with normal and obese BMI category breast cancer patients in the ER and PR-/ HER2+ subgroup. Recently, two studies evaluated the correlation between BMI and disease-free survival in HER2-positive breast cancer patients [11, 37]. One study reported that obesity decreases survival compared with normal weight [11], but the second study reported conflicting results [37]. Because these studies were analyzed only in the context of obese versus non-obese HER2positive patients, including ER and/or PR positive and ER and PR negative breast cancer patients in the small sample size, these studies have not demonstrated whether an underweight BMI is associated with an increased risk of mortality relative to normal weight in the ER and PR-/ HER2+ subgroup. The relationship between underweight BMI and decreased survival might be at least partly explained by the presence of circulating tumor cells (CTCs) in the peripheral blood of breast cancer patients. CTCs that have detached from the primary tumor site may reach a secondary organ and lead to metastases [38]. Furthermore, alterations of the circulating immune cells may influence tumor progression and the efficacy of systemic antitumor treatments. Chronic undernutrition and micronutrient deficiency compromise the cytokine response and affect immune cell trafficking, which might affect the tumor-immune system interaction in other organs [39].

In the ER and/or PR+/HER2+ and ER and PR-/ HER2- subgroups, BMI categories did not exhibit a significant association with OS and BCSS. Similar to our finding, one study reported that obesity was not associated with decreased survival in patients with triple-negative breast cancer [40]. Because the impact on BMI and breast cancer outcomes was masked by the effect of the ER and/or PR+/HER2- and ER and PR-/HER2+ subgroups, a weaker association between BMI and poor outcomes may exist in patients in the ER and/or PR+/HER2+ and ER and PR-/HER2- subgroup.

Our study has several strengths and limitations. The main strength of our study is its inclusion of a large sample
(4468 deaths from any cause, 2824 deaths from breast cancer among 41,021 breast cancer patients), permitting a detailed examination across multiple BMI categories during the long follow-up period. Furthermore, our study is the first to investigate the prognostic significance of BMI in four different breast cancer subtypes. However, our study was limited by the information available in the KBCR database. First, registered patients in the KBCR database were heterogeneous with respect to breast cancer stage, IHC staining results, and presence of comorbidities. Additionally, the essential registry data (BMI and HR expression) were only available for $62.84 \%$ of invasive breast cancer patients in the KBCR database. Therefore, the possibility of selection bias remains. Second, although total sample size is larger compared with previous studies, the sample size of underweight and obese BMI subjects is small to draw a conclusion on the independent effect of BMI. Finally, the ethnic homogeneity of the KBCR database may limit the generalizability of our finding to other racial and ethnic groups.

\section{Conclusions}

In conclusion, our results indicated a U-shaped relationship between BMI at diagnosis and poor OS and BCSS among all breast cancer patients, with the lowest risk observed among breast cancer patients with normal BMIs $\left(18.5-24.9 \mathrm{~kg} / \mathrm{m}^{2}\right)$. Among breast cancer patients with ER and/or PR+/HER2- tumors, obese individuals exhibit significantly poorer OS and BCSS, whereas among those with ER and PR-/HER2+ tumors, underweight patients exhibit significantly poorer OS and BCSS compared with breast cancer patients with normal BMIs. Although obesity and underweight BMI at diagnosis are poor prognostic factors in pooled breast cancer samples, BMI at diagnosis exhibited a different impact on breast cancer prognosis in specific breast cancer subtypes. Therefore, BMI at diagnosis and breast cancer subtype should be considered simultaneously in various treatment decision processes and surveillance schedules.

\section{Abbreviations}

HR: Hormone receptor; HER2: Human epidermal growth factor receptor 2; OS: Overall survival; BCSS: Breast cancer-specific survival; ER: Estrogen receptor; PR: Progesterone receptor; IHC: Immunohistochemistry; FISH: Fluorescence in situ hybridization; HR: Hazard ratio; Cl: Confidence interval.

\section{Competing interests}

The authors declare that they have no competing interests.

\section{Authors' contributions}

YW Jeon, YJ Suh participated in the design of the study. YW Jeon, YJ Suh participated in the statistical analysis and interpretation of the data. YJ Suh has been involved in revising it critically for important intellectual content. YW Jeon drafted the manuscript. YW Jeon, SH Kang, MH park, WS Lim, SH Cho and YJ Suh participated in the collection of the data. All authors read and approved the final manuscript. 


\section{Acknowledgements}

Statistical analysis support was provided by the Catholic Research Coordinating Center of the Korea Health 21 R\&D Project (A070001), Ministry of Health \& Welfare, Republic of Korea.

No funds were received in support of this study.

\section{Author details}

${ }^{1}$ Department of Surgery, St. Vincent's Hospital, College of Medicine, The Catholic University, 93 Joongboo-Daero Paldal-gu, Suwon 442-723, Kyunggi-do, Republic of Korea. ${ }^{2}$ Department of Surgery, Yeungnam University College of Medicine, 170 Hyunchung-ro, Nam-gu, Deagu 705-703, Republic of Korea. ${ }^{3}$ Department of Surgery, Chonnam National University Medical School, 160 Baekseo-ro, Dong-gu, Gwangju 501-746, Republic of Korea. ${ }^{4}$ Department of Surgery, Ewha Womans University, Mokdong hospital, 1071, Anyangcheon-ro, Yangcheon-gu, Seoul 158-710, Republic of Korea. ${ }^{5}$ Department of Surgery, Dong-A University College of Medicine, 26, Daesingongwon-ro, Seo-gu, Busan 602-715, Republic of Korea.

\section{Received: 14 October 2014 Accepted: 30 October 2015} Published online: 06 November 2015

\section{References}

1. Chang S, Alderfer JR, Asmar L, Buzdar AU. Inflammatory breast cancer survival: the role of obesity and menopausal status at diagnosis. Breast Cancer Res Treat. 2000;64:157-63.

2. Berclaz G, Li S, Price KN, Coates AS, Castiglione-Gertsch M, Rudenstam CM, et al. Body mass index as a prognostic feature in operable breast cancer: the International Breast Cancer Study Group experience. Ann Oncol. 2004; 15:875-84

3. Loi S, Milne RL, Friedlander ML, McCredie MR, Giles GG, Hopper JL, et al. Obesity and outcomes in premenopausal and postmenopausal breast cancer. Cancer Epidemiol Biomarkers Prev. 2005;14:1686-91.

4. Dignam JJ, Wieand K, Johnson KA, Raich P, Anderson SJ, Somkin C, et al. Effects of obesity and race on prognosis in lymph node-negative, estrogen receptor-negative breast cancer. Breast Cancer Res Treat. 2006;97:245-54.

5. Demirkan B, Alacacioglu A, Yilmaz U. Relation of body mass index (BMI) to disease free (DFS) and distant disease free survivals (DDFS) among Turkish women with operable breast carcinoma. Jpn J Clin Oncol. 2007;37:256-65.

6. Vitolins MZ, Kimmick GG, Case LD. BMI influences prognosis following surgery and adjuvant chemotherapy for lymph node positive breast cancer. Breast J. 2008;14:357-65.

7. Caan BJ, Kwan ML, Hartzell G, Castillo A, Slattery ML, Sternfeld B, et al. Pre-diagnosis body mass index, post-diagnosis weight change, and prognosis among women with early stage breast cancer. Cancer Causes Control. 2008;19:1319-28.

8. de Azambuja E, McCaskill-Stevens W, Francis P, Quinaux E, Crown JP, Vicente $M$, et al. The effect of body mass index on overall and disease-free survival in node-positive breast cancer patients treated with docetaxel and doxorubicincontaining adjuvant chemotherapy: the experience of the BIG 02-98 trial. Breast Cancer Res Treat. 2010;119:145-53.

9. Chen X, Lu W, Zheng W, Gu K, Chen Z, Zheng Y, et al. Obesity and weight change in relation to breast cancer survival. Breast Cancer Res Treat. 2010;122:823-33.

10. Keegan TH, Milne RL, Andrulis IL, Chang ET, Sangaramoorthy M, Phillips KA et al. Past recreational physical activity, body size, and all-cause mortality following breast cancer diagnosis: results from the Breast Cancer Family Registry. Breast Cancer Res Treat. 2010;123:531-42.

11. Sparano JA, Wang M, Zhao F, Stearns V, Martino S, Ligibel JA, et al. Obesity at diagnosis is associated with inferior outcomes in hormone receptor-positive operable breast cancer. Cancer. 2012;118:5937-46.

12. Sestak I, Distler W, Forbes JF, Dowsett M, Howell A, Cuzick J. Effect of body mass index on recurrences in tamoxifen and anastrozole treated women: an exploratory analysis from the ATAC trial. J Clin Oncol. 2010;28:3411-5.

13. Kwan ML, Chen WY, Kroenke CH, Weltzien EK, Beasley JM, Nechuta SJ, et al Pre-diagnosis body mass index and survival after breast cancer in the After Breast Cancer Pooling Project. Breast Cancer Res Treat. 2012;132:729-39.

14. Conroy SM, Maskarinec G, Wilkens LR, White KK, Henderson BE, Kolonel LN. Obesity and breast cancer survival in ethnically diverse postmenopausal women: the Multiethnic Cohort Study. Breast Cancer Res Treat. 2011:129:565-74.

15. Lu Y, Ma H, Malone KE, Norman SA, Sullivan-Halley J, Strom BL, et al. Obesity and survival among black women and white women 35 to 64 years of age at diagnosis with invasive breast cancer. J Clin Oncol. 2011;29:3358-65.
16. Ewertz M, Jensen MB, Gunnarsdóttir KÁ, Højris I, Jakobsen EH, Nielsen D, et al. Effect of obesity on prognosis after early-stage breast cancer. J Clin Oncol. 2011;29:25-31.

17. Kawai M, Minami Y, Nishino Y, Fukamachi K, Ohuchi N, Kakugawa Y. Body mass index and survival after breast cancer diagnosis in Japanese women. BMC Cancer. 2012;12:149.

18. Moon HG, Han W, Noh DY. Underweight and breast cancer recurrence and death: a report from the Korean Breast Cancer Society. J Clin Oncol. 2009;27:5899-905.

19. Chauvet B, Reynaud-Bougnoux A, Calais G, Panel N, Lansac J, Bougnoux P, et al. Prognostic significance of breast relapse after conservative treatment in node-negative early breast cancer. Int J Radiat Oncol Biol Phys. 1990;19:1125-30.

20. Marret $H$, Perrotin F, Bougnoux P, Descamps P, Hubert B, Lefranc T, et al. Low body mass index is an independent predictive factor of local recurrence after conservative treatment for breast cancer. Breast Cancer Res Treat. 2001;66:17-23.

21. Enger SM, Bernstein L. Exercise activity, body size and premenopausal breast cancer survival. Br J Cancer. 2004;90:2138-41.

22. Carmichael AR, Bendall S, Lockerbie L, Prescott RJ, Bates T. Does obesity compromise survival in women with breast cancer? Breast. 2004;13:93-6.

23. Niraula S, Ocana A, Ennis M, Goodwin PJ. Body size and breast cancer prognosis in relation to hormone receptor and menopausal status: a meta-analysis. Breast Cancer Res Treat. 2012;134:769-81.

24. Ladoire S, Dalban C, Roché H, Spielmann M, Fumoleau P, Levy C, et al. Effect of obesity on disease-free and overall survival in node-positive breast cancer patients in a large French population: a pooled analysis of two randomised trials. Eur J Cancer. 2014;50:506-16.

25. Perou CM, Sørlie T, Eisen MB, van de Rijn M, Jeffrey SS, Rees CA, et al. Molecular portraits of human breast tumours. Nature. 2000;406:747-52.

26. Sørlie T, Perou CM, Tibshirani R, Aas T, Geisler S, Johnsen H, et al. Gene expression patterns of breast carcinomas distinguish tumor subclasses with clinical implications. Proc Natl Acad Sci U S A. 2001;98:10869-74.

27. World Health Organization. Western Pacific Region, IASO International Association for the Study of Obesity, International Obesity Task Force: The Asia Pacific perspective: Redefining obesity and its treatment. Australia: Health Communications Australia; 2000.

28. Deurenberg-Yap M, Chew SK, Deurenberg P. Elevated body fat percentage and cardiovascular risks at low body mass index levels among Singaporean Chinese, Malays and Indians. Obes Rev. 2002;3:209-15.

29. Gurrici S, Hartriyanti Y, Hautvast JG, Deurenberg P. Relationship between body fat and body mass index: differences between Indonesians and Dutch Caucasians. Eur J Clin Nutr. 1998:52:779-83.

30. Jeon YW, Choi JE, Park HK, Kim KS, Lee JY, Suh YJ. Impact of local surgical treatment on survival in young women with T1 breast cancer: long-term results of a population-based cohort. Breast Cancer Res Treat. 2013;138:475-84.

31. Edge SB, Byrd DR, Compton CC, Fritz AG, Greene FL, Trotti A. AJCC Cancer Staging Manual. 7th ed. New York: Springer; 2010.

32. BMl classification [http://apps.who.int/bmi/index.jsp?introPage=intro_3.html]. Accessed date: 5 November 2015.

33. Jang M, Berry D. Overweight, obesity, and metabolic syndrome in adults and children in South Korea: a review of the literature. Clin Nurs Res. 2011;20:276-91.

34. Cleary MP, Grossmann ME. Minireview: obesity and breast cancer: the estrogen connection. Endocrinology. 2009;150:2537-42.

35. Papa V, Belfiore A. Insulin receptors in breast cancer: biological and clinical role. J Endocrinol Invest. 1996;19:324-33.

36. Rose DP, Gilhooly EM, Nixon DW. Adverse effects of obesity on breast cancer prognosis, and the biological actions of leptin (review). Int J Oncol. 2002;21:1285-92

37. Crozier JA, Moreno-Aspitia A, Ballman KV, Dueck AC, Pockaj BA, Perez EA Effect of body mass index on tumor characteristics and disease-free survival in patients from the HER2-positive adjuvant trastuzumab trial N9831. Cancer. 2013;119:2447-54.

38. Paget $\mathrm{S}$. The distribution of secondary growths in cancer of the breast 1889. Cancer Metastasis Rev. 1989:8:98-101.

39. Cunningham-Rundles S, McNeeley DF, Moon A. Mechanisms of nutrient modulation of the immune response. J Allergy Clin Immunol. 2005;115:1119-28. quiz 1129.

40. Mowad R, Chu QD, Li BD, Burton GV, Ampil FL, Kim RH. Does obesity have an effect on outcomes in triple-negative breast cancer? J Surg Res. 2013;184:253-9. 